\title{
Presencia de Hemoparásitos en Tortugas M otelo (Chelonoides denticulata) (Linnaeus, 1766) Comercializadas en el Mercado de Belén, Iquitos, Perú
}

\author{
Presence of Blood Parasites in the Yellow-footed Tortoise (Chelonoides \\ denticulata) (Linnaeus, 1766) Sold at the Belen Market, Iquitos, Peru
}
Luis Batalla L.1,6, Eva Casas A.,7, Rosa Julca ${ }^{1}$, Nofre Sánchez², Roxana Angelats ${ }^{3}$, Gianmarco Rojas ${ }^{4}$, Jorge Cárdenas ${ }^{5}$

\section{Resumen}

\begin{abstract}
El objetivo del presente estudio fue identificar y determinar la presencia de hemoparásitos en tortugas motelo comercializadas en el mercado de Belén, Iquitos, Perú, así como su asociación con las variables sexo, peso, hematocrito y parasitemia. Se colectaron muestras de sangre de 47 tortugas en julio de 2010. Se utilizaron las técnicas de frotis sanguíneo delgado con Giemsa, microcapilar de Woo y técnica de capa blanca o 'Buffy coat' para la identificación de los hemoparásitos. Se encontró la presencia de Haemogregarina sp, con una frecuencia de $74.5 \%$, siendo de $68.8 \%$ en hembras y $86.7 \%$ en machos. Se encontraron gamontes maduros $(11.09 \pm 1.42 \mu \mathrm{m}$ de longitud x $5.82 \pm 0.76$ $\mu \mathrm{m}$ de ancho) e inmaduros $(14.85 \pm 0.69 \mu \mathrm{m} \times 3.26 \pm 0.41 \mu \mathrm{m})$. No se encontró diferencia significativa entre la presencia del parásito con las variables sexo, peso, hematocrito y nivel de parasitemia.
\end{abstract}

Palabras clave: Chelonoides denticulata, tortuga patas amarillas, hemoparásitos, hemogregarina

\footnotetext{
${ }^{1}$ Laboratorio de Microbiología y Parasitología Veterinaria, Facultad de Medicina Veterinaria, Universidad Nacional Mayor de San Marcos, Lima, Perú

${ }^{2}$ Estación Experimental del Centro de Investigación IVITA-Iquitos, Universidad Nacional Mayor de San Marcos, Iquitos, Perú

${ }^{3}$ Agrovet Market Animal Health, Lima, Perú

${ }^{4}$ Parque Zoológico Huachipa, Lima, Perú

${ }^{5}$ Global Health Initiative, Wabash College, Indiana, EEUU

${ }^{6}$ E-mail: luisbatalla17@gmail.com

${ }^{7}$ E-mail: evacasas99@gmail.com
}

Recibido: 8 de octubre de 2014

Aceptado para publicación: 30 de marzo de 2015 
The aim of this study was to identify and determine the presence of blood parasites in yellow-footed tortoises sold in the market of Belen, Iquitos, Peru and their association with sex, body weight, hematocrit and parasitemia. Blood samples were collected from 47 tortoises in July 2010. The thin blood smears with Giemsa, the microcapillary Woo technique and the Buffy coat technique were used for the identification of the haemoparasites. The results showed the presence of Haemogregarina sp in $74.5 \%$ of the samples, being $68.8 \%$ in females and $86.7 \%$ in males. There were mature gamonts $(11.09 \pm 1.42 \mu \mathrm{m}$ length $\mathrm{x} 5.82 \pm 0.76 \mu \mathrm{m}$ wide) and inmature gamonts $(14.85 \pm 0.69 \mu \mathrm{m} \times 3.26 \pm 0.41 \mu \mathrm{m})$. There were no significant differences between the presence of the parasite and the variables sex, weight, hematocrit and parasitemia level.

Keywords: Chelonoides denticulata, yellow-footed tortoise, hemoparasites, haemogregarine

\section{INTRODUCCIÓN}

Chelonoides denticulata (Linnaeus, 1766), conocida como «tortuga motelo», es una de las cuatro especies de Testudíneos que se encuentran distribuidas en Sudamérica. Diversos estudios señalan a las tortugas como hospedadoras de diversos agentes parasitarios, entre ellos los hemoparásitos. Estos últimos se caracterizan por su característica de forma elongada, ligeramente curvados, extremos redondeados pero sin curvatura, con núcleo denso, y bordes bien definidos, principalmente en su forma adulta.

Estudios realizados en tortugas terrestres demuestran prevalencias de hemoparásitos de 35 a $72 \%$ (Siroký et al., 2004; Cook et al., 2009). En Chelonoides denticulata solo se ha reportado Haemoproteus geochelonis (Lainson $\mathrm{y}$ Naiff, 1998), en tanto que en tortugas acuáticas de América del Sur se ha encontrado Haemogregarina sp en Podocnemis expansa (Nascimento, 2008) y Phrynops geoffroanus (De campos, 2002) y en otros lugares Haemogregarina chelandi en Emydura signata (Jakes et al., 2001), Haemogregarina balli en Emys orbicularis (Mihalca y Achelartei, 2002) y Haemogregarina fitzsimonsi en Stigmochelys pardalis (Cook et al., 2009).
Los hemoparásitos en tortugas aparentemente están bien adaptados a su hospedero, causando poca o ninguna enfermedad, pero podrían causar patologías inflamatorias clínicas en hospederos no naturales, entre ellas, hepatitis, pancreatitis y esplenitis (Pessoa et al., 1974; Wozniak y Telford, 1991). No hay muchos reportes de lesiones de hemoparásitos en tortugas como en otros reptiles, pero en infecciones con alta parasitemia se puede observar un cuadro de anemia hemolítica (Campbell, 1996).

Los hemoparásitos provocan una disminución en el crecimiento y las hembras infectadas disminuyen el rendimiento reproductivo (Madsen et al., 2005). Asimismo, en situaciones desfavorables que afectan la inmunidad y posibilitan el ingreso de otros agentes patógenos, puede producir lesiones por la obstrucción de capilares debido al aumento de la carga parasitaria, ocasionando trombosis y necrosis tisular (Frye, 1991). A nivel celular, se menciona una amplia gama de lesiones que va desde infiltración celular muy leve hasta necrosis (Gardiner et al., 1988).

En el Perú no se ha registrado la presencia de Trypanosoma sp en tortugas terrestres. Es así que dada la escasa información sobre presencia de hemoparásitos en 
tortugas de la Amazonía peruana, el presente estudio tuvo como objetivo identificar y describir los hemoparásitos presentes en tortugas motelo (Chelonoides denticulata) procedentes de la zona de Loreto, Perú.

\section{Materiales y Métodos}

\section{Lugar de Estudio}

El estudio se realizó en el mercado de abastos del distrito de Belén, Iquitos, departamento de Loreto, Perú. Iquitos se encuentra ubicado en la selva baja, a $106 \mathrm{msnm}$, con temperaturas de 20 a $36{ }^{\circ} \mathrm{C}$, humedad relativa de 68 a $93 \%$ y precipitaciones anuales de 2000 a 3000 mm (SENAMHI, 2010).

El procesamiento de las muestras se realizó en el laboratorio de la Estación Experimental IVITA-Iquitos y en el Laboratorio de Parasitología de la Facultad de Medicina Veterinaria, Lima, ambos de la Universidad Nacional Mayor de San Marcos, así como en el Laboratorio de Ixodides, Departamento de Entomología, Instituto Oswaldo CruzFiocruz, Brasil, donde se confirmó la especie de la garrapata.

\section{Animales y Muestras}

Se recolectaron muestras de sangre (4$5 \mathrm{ml}$ ) por punción en la vena braquial (Owens y Ruiz, 1980) en tubos estériles de vidrio al vacío con anticoagulante de heparina-litio. Se trabajó con 47 tortugas motelo, sin distinción de sexo y edad, en julio de 2010, que se encontraban a la venta en el mercado de Belén, Iquitos. Las tortugas procedían de diversas zonas del departamento de Loreto, desde donde eran capturadas y trasladadas al mercado de Belén para la comercialización de su carne y huevos.

En las muestras de sangre se utilizó la técnica de frotis sanguíneo delgado y coloración Giemsa. Los frotis se observaron al microscopio con objetivos de 40X o 100X con aceite de inmersión.
En forma paralela, se utilizó la técnica del Microcapilar de Woo (FAO, 1998), observando la capa leucocitaria al microscopio con el objetivo de 10X o 20X para detectar la presencia de hemoparásitos. Asimismo, para determinar la morfología de los tripanosomas se utilizó la técnica de capa blanca o 'Buffy coat', donde el microcapilar utilizado en la técnica de Woo se rompe a $1 \mathrm{~mm}$ por debajo de la capa blanca y el contenido se deposita en una lámina, donde se hace una extensión, se fija con metanol por 5 min y se colorea con Giemsa por 30 min. La lámina se observa al microscopio con aceite de inmersión a $100 X$.

La identificación se hizo siguiendo las características morfológicas de los hemoparásitos (Mackerras, 1961; Pessoa et al., 1972; Mihalca y Achelartei, 2002; Moço et al., 2002). La intensidad de parasitemia (porcentaje de eritrocitos parasitados) se calculó según el método de Siroký et al. (2007) contando 100 glóbulos rojos por campo en un total de 100 campos. Asimismo, la muestra es considerada positiva cuando se encuentra un hemoparásito, así sea en un campo.

\section{Análisis de Datos}

Se calculó la frecuencia de hemoparásitos con sus respectivos intervalos de confianza al 95\% de confianza a partir de la proporción de muestras positivas. La variable sexo fue analizada mediante la prueba de Chi Cuadrado y la parasitemia mediante la prueba de U Mann Whitney. Las variables peso $(\mathrm{kg})$, hematocrito $(\mathrm{Ht})$ y hemoparásitos fueron analizadas con la prueba de «t» Student. Las variables sexo y $\mathrm{Ht}$ fueron expresados con una media, desviación estándar y rango. Los datos fueron analizados con el paquete estadístico SPSS ${ }^{\circledR}$.

\section{Resultados}

El único tipo de hemoparásitos que se encontró fue Haemogregarina sp, no hallándose la presencia de tripanosomas ni otros 
Cuadro 1. Hematocrito y peso vivo en 47 tortugas motelo (Chelonoides denticulata), infectadas o no infectadas por Haemogregarina sp, muestreadas en el mercado de Belén, Iquitos, Perú (2010)

\begin{tabular}{lccc}
\hline \multirow{2}{*}{ Variable } & \multicolumn{2}{c}{ Infección con Haemogregarina sp } & \multirow{2}{*}{$\mathrm{p}$} \\
\cline { 2 - 3 } & Negativas (\%) & Positivas (\%) & \\
\hline Hematocrito & $22.3 \pm 8.4$ & $19.7 \pm 7.2$ & 0.31 \\
Peso & $5.5 \pm 1.2$ & $5.4 \pm 1.6$ & 0.77 \\
\hline
\end{tabular}

Cuadro 2. Parasitemia de Haemogregarina sp en 47 tortugas motelo (Chelonoides denticulata) muestreadas en el mercado de Belén, Iquitos, Perú (2010)

\begin{tabular}{lccccc}
\hline \multirow{2}{*}{ Sexo } & \multirow{2}{*}{ Total $(\mathrm{n})$} & \multirow{2}{*}{ Positivos $(\mathrm{n})$} & \multicolumn{2}{c}{ Parasitemia } & \multirow{2}{*}{$\mathrm{p}$} \\
\cline { 4 - 5 } & & & Media $\pm \mathrm{IC}^{1}(\%)$ & Rango & \\
\hline Hembra & 32 & 22 & $0.90 \pm 0.13$ & $0.10-5.30$ & \multirow{2}{*}{0.35} \\
Macho & 15 & 13 & $0.60 \pm 0.51$ & $0.10-1.64$ & \\
\hline Total & 47 & $35(74.5 \%)$ & 0.80 & $0.1-5.30$ & \\
\hline
\end{tabular}

${ }^{1}$ Intervalo de confianza del $95 \%$

flagelados o filaroides. La frecuencia de Haemogregarina sp mediante la técnica de frotis sanguíneo delgado fue de $74.5 \%$ (35/47), siendo de $68.8 \%$ (22/32) en hembras y $86.7 \%(13 / 15)$ en machos, aunque sin diferencia estadística.

No se encontró efecto significativo en relación a las variables peso, hematocrito (Cuadro 1), sexo y parasitemia (Cuadro 2) con relación a la presencia de Haemogregarina $\mathrm{sp}$.

La lectura de las láminas mostró gamontes de Haemogregarina sp en base a sus características morfológicas: forma elongada, ligeramente curvados, extremos redondeados pero sin curvatura, con núcleo denso, bordes bien definidos y, en algunos de los casos, el parásito se encontraba encapsulado, principalmente en su forma adulta.

Así mismo, se observó formas de gamontes inmaduros de Haemogregarina sp, que se encontraban cerca al núcleo del eritrocito o alejados; la mayoría delgados, alargados y curvados, pudiendo mantener la posición del núcleo en el centro aunque generalmente se encuentra más cerca a uno de los dos polos (Fig. 1). Tienen una forma de banano, aunque es llamado también silla de montar, pues no deforma al eritrocito. Las medidas de los gamontes inmaduros fueron $14.85 \pm 0.69 \mu \mathrm{m}(13.78 \mathrm{a} 15.95 \mu \mathrm{m})$ de longitud por $3.26 \pm 0.41 \mu \mathrm{m}(2.90 \mathrm{a} 4.35 \mu \mathrm{m}) \mathrm{de}$ ancho $(n=16)$. 

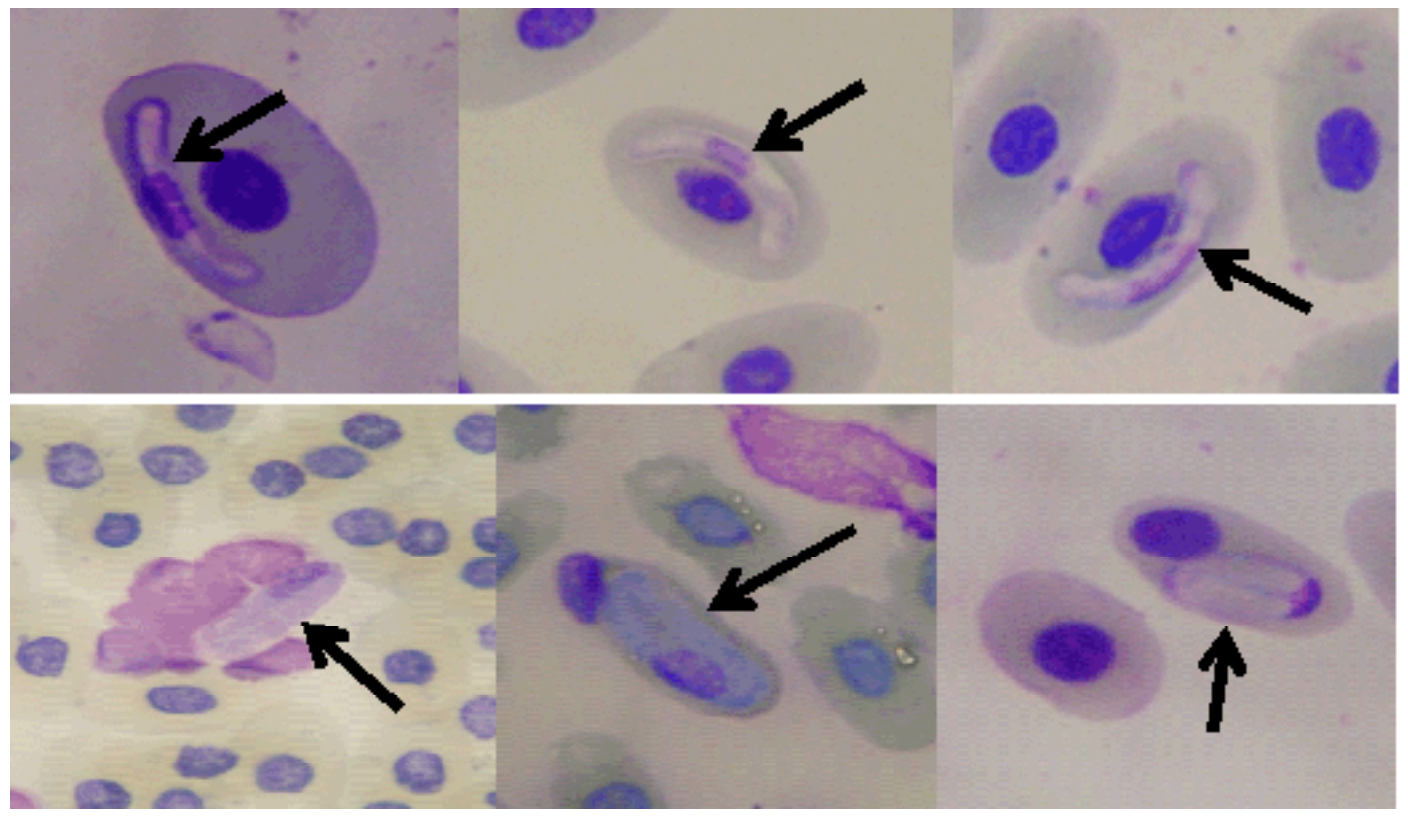

Figura 1. Haemogregarina sp (flechas) en tortugas motelo (Geochelone denticulata). En el panel superior se observan gamontes inmaduros intraeritrocitarios, alargados y curvados con bordes redondeados. En el panel inferior se observan gamontes maduros, elongados con desplazamiento del núcleo del parásito hacia un extremo, causando desplazamiento del núcleo y distorsión del eritrocito. Tinción Giemsa 1000X

En el caso de gamontes maduros de Haemogregarina sp, cada uno ocupaba individualmente un eritrocito, aunque hubo casos con dos gamontes en un mismo eritrocito. Los gamontes maduros son elongados, a veces con un polo opuesto y amplio con una pequeña cola recurvada, caracterizada por el desplazamiento del núcleo del hemoparásito hacia uno de los polos (Fig. 1). En esta etapa los gamontes maduros causaron distorsión del eritrocito e inclusive la lisis. Las medidas para los gamontes maduros fueron $11.09 \pm 1.42$ $\mu \mathrm{m}(9.82 \mathrm{a} 17.73 \mu \mathrm{m})$ de longitud por $5.82 \pm$ $0.76 \mu \mathrm{m}$ (4.73 a $8.03 \mu \mathrm{m})$ de ancho $(\mathrm{n}=29)$.

\section{Discusión}

Danilewsky (1885) fue el primero en describir la Haemogregarina en la tortuga acuática Emys orbicularis, encontrando gamontes de $32.0 \pm 1.9 \mu \mathrm{m}$ de longitud y de $4.2 \pm 0.7 \mu \mathrm{m}$ de ancho; Jakes et al. (2001) reporta gamontes maduros de 15.5 por $7.1 \mu \mathrm{m}$ ancho y gamontes inmaduros de 9.2 por 4.6 $\mu \mathrm{m}$ en la tortuga acuática Emydura signata, en tanto que Siroký et al. (2004) reporta gamontes maduros de 12.5 por $5 \mu \mathrm{m}$ y gamontes inmaduros de 8.5 por $5 \mu \mathrm{m}$ en una Testudo marginata. Por otro lado, Cook et al. (2009) reporta gamontes inmaduros de 17.8 por $2.3 \mu \mathrm{m}$ y gamontes maduros de 17.5 por $3.9 \mu \mathrm{m}$ en una Chersina angulata.

Campell (1996) y Telford et al. (2009) indican que en la mayoría de tortugas semiacuáticas y tortugas terrestres predomina el género Haemogregarina. La especie de Haemogregarina, por el tamaño de gamontes en el presente estudio, podría pertenecer a una nueva especie, debido a las variaciones en tamaño y forma (Pessoa $e t$ al., 1974). 
Mackerras (1961) y Pessoa et al. (1972) señalan que cuando se encuentra hemoparásitos en tortugas terrestres donde se desconoce el ciclo de vida del vector, esquizontes en tejidos y no hay estudios previos en esa zona, se puede adoptar el género Haemogregarina (sensu lato) sp hasta que nuevos datos sean descritos para su clasificación. Por lo tanto, con estos antecedentes, se puede afirmar que el hemoparásito encontrado en la presente investigación se asemeja al género Haemogregarina; sin embargo, es necesario la verificación del desarrollo y ciclo de vida del hospedador invertebrado para su correcta identificación (Desser et al., 1995; Smith, 1996; Jakes et al., 2003).

El $74.5 \%$ de frecuencia de Haemogregarina sp del presente estudio es similar a otros reportes: $72 \%$ de $H$. mauritanica en Testudo marginata (Siroký et al., 2004), $100 \%$ de infección de $H$. chelandi en Emydura signata y $77 \%$ de $H$. chelandi en Elseya latisternum (Jakes et al., 2001), y $100 \%$ de infección de H. balli en Emys orbicularis (Mihalca y Achelartei, 2002); en tanto que Cook et al. (2009) reportan una prevalencia de $35 \%$ de $H$. fitzsimonsi en Stigmochelys pardalis. Asimismo, los resultados encontrados señalan que el sexo no es un factor relevante para la presencia de Haemogregarina sp.

La parasitemia encontrada fue de $0.8 \%$, con rangos de 0.1 a $5.30 \%$, sin diferencia significativa entre sexos, a diferencia del estudio de Telford et al. (2009) donde reportan parasitemias inferiores en Macrochelys temminckii. Por otro lado, Mihalca y Achelartei (2002) reporta parasitemias de 0.8 a $2.2 \%$ en Emys orbicularis y Jakes et al. (2001) reporta rangos de 1 a 2\% en Emydura signata, en tanto que Cook et al. (2009) reporta rangos 2.5 a $2.6 \%$ en $S$. pardalis.

Campbell (1996) señala que encontrar hemoparásitos en tortugas terrestres es raro o no han sido reportados. Jakes et al. (2001) reportan Trypanosoma chelodinae y Haemoproteus chelodinae en Emydura signata y E. latisternum. Lainson y Naiff (1998) describen Haemoproteus geochelonis en Geochelone denticulata capturada en selva alta en el estado de Pará, Brasil, sin encontrar el vector; mientras que DeGiusti et al. (1973) señalan que sería la mosca del tabano Chrysops Callidus en la tortuga $C$. picta. Se conoce que los mayores casos de hemoparásitos en tortugas ocurren en las especies acuáticas y no tanto en las terrestres debido a que el medio favorece el ciclo de vida. Es posible que en el presente estudio no se encontraron otras formas de hemoparásitos debido a que las condiciones ambientales para el ciclo de vida no estuvieron presentes.

\section{Conclusiones}

Se encontró una presencia de $74.5 \%$ de Haemogregarina sp (35/47), mediante frotis sanguíneo y coloración Giemsa, en tortugas motelo (Geochelone denticulata) comercializadas en el mercado de Belén.

\section{Literatura Citada}

1. Campbell T. 1996. Hemoparasites. In: Reptile medicine \& surgery. Philadelphia, USA: WB Saunders. p 379-381.

2. Cook CA, Smit NJ, Davies AJ. 2009. A redescription of Haemogregarina fitzsimonsi Dias, 1953 and some comments on Haemogregarina parvula Dias, 1953 (Adeleorina: Haemogregarinidae) from southern African tortoises (Cryptodira: Testudinidae), with new host data and distribution records. Folia Parasitol 56: 173-179.

3. Danilewsky VI. 1885. De Hamatozoen der Kaltbluter. Arch. Mikr Anat 24: 588598.

4. De Campos VB. 2002. Hematologia, bioquímica do sangre, parasitologia, microbiologia, algas epizoárias e histopatologia de Phrynops geoffroanus 
(Schweigger, 1812) (Testudinata, Chelidae), expostos a diferentes influencias antrópicas no rio Uberabihna, Minas Gerias. Tesis de Médico Veterinario. São Carlos, Brazil: Univ Federal de São Carlos. 220 p.

5. DeGiusti DL, Sterling CR, Dobrzechowsky D. 1973. Transmission of the chelonian haemoproteid Haemoproteus metchnikovi by a tabanid fly Chrysops callidus. Nature 242: 50-51. doi: 10.1038/242050a0

6. Desser SS, Hong H, Martin DS. 1995. The life history, ultrastructure, and experimental transmission of Hepatozoon catesbianae n. comb., an apicomplexan parasite of the bullfrog, Rana catesbeiana and the mosquito, Culex territans in Algonquin Park, Ontario. J Parasitol 81: 212-222.

7. [FAO] Food and Agriculture Organization of the United Nations. 1998. A field guide for the diagnosis, treatment and prevention of African animal trypanosomosis. [Internet], [20 septiembre 2011]. Disponible en: http:// www.fao.org/docrep/006/X0413E/ X0413E04.htm\#ch3

8. Frye F. 1991. Biomedical and surgical aspects of captive reptile husbandry. $2^{\text {nd }}$ ed. Philadelphia, USA: Krieger Publ. 712 p.

9. Gardiner CH, Fayer R, Dubey JP. 1988. An atlas of protozoan parasites in animal tissues. USA: Department of Agriculture, Agricultural Research Service. N. ${ }^{\circ} 651.83 \mathrm{p}$.

10. Jakes KA, O'Donoghue, Munro M, Adlard R. 2001. Hemoprotozoa of freshwater turtles in Queensland. J Wildlife Dis 37: 12-19. doi: 10.7589/00903558-37.1.12

11. Jakes KA, O'Donoghue, Cameron S. 2003. Phylogenetic relationship of Hepatozoon (Hemogregarina) boigae, Hepatozoon sp., Haemogregarina clelandi and Haemoproteus chelodina form Australian reptilies to other Apicomplexa based on cladistic analyses of ultrastructuraland lifecycle characters. Parasitolology 126: 555-559.

12. Lainson $R, \quad$ Naiff RD. 1998. Haemoproteus (Apicomplexa: Haemoproteidae) of tortoises and turtles. Proc Biol Sci 265: 941-949. doi: 10.1098/ rspb.1998.0382

13. Mackerras MJ. 1961. The haematozoa of Australian reptiles. Aust J Zool 9: 61122. doi: 10.1071/ZO9610061

14. Madsen T, Ujvari B, Olsson M. 2005. Old pythons stay fit; effects of haematozoan infections on life history traits of a large tropical predator. Oecologia 142: 407-412. doi: 10.1007/ s00442-004-1742-9

15. Mihalca DA, Achelartei PP. 2002. Hemoparasites of the genus Haemogregarina in a population of European pond turtles (Emys orbicularis) from Dragasani, Valcea county, Romania. Sci Parasitol 2: 22-27.

16. Moço TC, Odwyer LH, Vilela FC, Barrella TC, Silva RJ. 2002. Morphologic and morphometric analysis of Hepatozoon spp (Apicomplexa , Hepatozoidae) of snakes. Mem Inst Oswaldo Cruz 97: 1169-1176. doi: 10.1590/S0074-02762002000800019

17. Nascimento FA, 2008. Aspectos alimentares e do parasitismo em Podocnemis expansa de ambiente natural e cativeiro comercial no Estado do Tocantins. Tesis de Ciências do Ambiente. Palmas, Brasil: Universidade Federal do Tocantins. $163 \mathrm{p}$.

18. Owens D, Ruiz G, 1980. New methods of obtaining blood and cerebrospinal fluid from marine turtles. Herpetol 36: 17-20.

19. Pessoa SB, Biasi P, Puorto G. 1974. Nota sobre a frequencia de hemoparasitas em serpentes do Brasil. Mem Inst Butantan 38: 69-118.

20. Pessoa SB, Biasi P, Souza D. 1972. Esporulacao do Hepatozoon caimani (Carini, 1909), parasita do jacare-e-papoamarelo: Caiman latirostris Daud, no Culex dolosus (L. Arribalzaga). Mem Inst Oswaldo Cruz 70: 379-383. 
21. [SENAMHI] Servicio Nacional de Metereologia e Hidrología del Perú. 2010. Datos históricos del clima-Loreto. Portal oficial [Internet]. [11 julio 2011]. Disponible en: http://www.senamhi.gob. pe/include_mapas/_dat_esta_tipo.php? estaciones $=000279$

22. Šroky P, Kamler M, Frye FL, Fictum P, Modry D. 2007. Endogenous development of Hemolivia mauritanica (Apicomplexa: Adeleina: Haemogregarinidae) in marginated tortoise Testudo marginata (Reptilia: Testudinidae): evidence from experimental infections. Folia Parasitol 54: 13-18.

23. Široky P, Kamler M, Modry D. 2004. Long-term occurrence of Hemolivia c.f. mauritanica (Apicomplexa: Adeleina: Haemogregarinidae) in captive Testudo marginata (Reptilia: Testudinidae: evidence for cyclic merogony? J
Parasitol 90: 1391-1393. doi: 10.1645/GE3306

24. Smith TG. 1996. The genus Hepatozoon (Apicomplexa: Adeleina). J Parasitol 82: 565-585.

25. Telford SR, Norton TM, Moler PE, Jensen JB. 2009. A new Haemogregarina species of the alligator snapping turtle, Macrochelys temminckii (Testudines: Chelydridae) in Georgia and Florida that produces macromeronts in circulating erythrocytes. J Parasitol 95: 208-214. doi: 10.1645/GE-1696.1

26. Wozniak EJ, Telford Jr. 1991. The fate of Hepatozoon species naturally infecting Florida black racers and watersnakes in potential mosquito and soft tick vectors, and histological evidence of pathogenicity in unnatural host species. J Parasitol 21: 511-516. doi: 10.1016/0020-7519(91)90055-C 